\title{
Dominant Mode Wave Impedance of Regular Polygonal Waveguides
}

\author{
Vyacheslav V. Komarov \\ Institute of Electronic and Mechanical Engineering, Yuri Gagarin State Technical University of Saratov, Saratov 410054, Russia \\ Correspondence should be addressed to Vyacheslav V. Komarov; vyacheslav.komarov@gmail.com
}

Received 25 October 2013; Revised 26 December 2013; Accepted 30 December 2013; Published 6 February 2014

Academic Editor: Chien-Jen Wang

Copyright ( 2014 Vyacheslav V. Komarov. This is an open access article distributed under the Creative Commons Attribution License, which permits unrestricted use, distribution, and reproduction in any medium, provided the original work is properly cited.

Polygonal metal waveguides are analyzed analytically and numerically. Classical equation for the wave impedance of arbitrary shaped waveguides is completed with approximate expression for the cutoff wavelength of the dominant mode. Proposed approach is tested with the help of 3D finite difference time domain models of microwave waveguides junctions. Obtained data are used for computer-aided design of microwave transition from coaxial line to cylindrical waveguide.

\section{Introduction}

Regular polygonal waveguides (RPW) with different number of side walls $(n)$ find application in microwave engineering as basic units of antennas [1], orthomode transducers [2], Tjunctions [3], mode transformers [4], heating devices [5], and power combiners [6]. Distribution of the TE- and TM-modes in such waveguides can be obtained from the Helmholtz equation solution with Neumann and Dirichlet boundary conditions on metal walls. But the exact analytic solution of this problem is possible only for the triangular $(n=3)$ [7] and square $(n=4)$ waveguides. Approximate analytical approaches based, for example, on perturbation method [8], method of analytical regularization [9], method of analogy [10], or some others [11] are also used for calculation of the eigenvalues and eigenfields of RPW. Some of these methods are restricted either by the waveguide shape [7] or by the mode type [11]. Numerical techniques implemented in commercial packages, HFSS, CST MWS, and MATLAB, are the alternative tools of modeling electromagnetic fields in RPW $[9,10,12]$.

Most of publications mentioned above are devoted to the computation of the cutoff wavenumbers of hollow RPW with $3 \leq n \leq 8$. Closed-form expressions for approximate calculation of the characteristic impedance, attenuation, and phase constant have been derived in [10], where a very simplified equation for wave impedance without any examples of its application is represented.

The objective of the present study was to check the applicability of metal waveguides theory to the modeling of wave impedance of RPW.

\section{Wave Impedance of RPW}

Electromagnetic characteristics of any RPW depend on the metal wall size $\left(a_{n}\right)$, which can be determined using another geometrical parameter-outer radius $\left(R_{n}\right)$ :

$$
a_{n}=2 R_{n} \sin \left(\frac{\pi}{n}\right) \text {. }
$$

As it is known, $a_{n}>R_{n}$, for $3 \leq n \leq 5 ; a_{6}=R_{6}$, while for the rest waveguides $a_{n}<R_{n}$ when $n \geq 7$ (Figure 1).

The wave impedance of the lowest TE-mode of any shaped metal waveguide:

$$
Z_{n}^{\mathrm{TE}}=\frac{k \eta}{\beta}=\frac{120 \pi}{\sqrt{1-\left(\lambda / \lambda_{c n}\right)^{2}}},
$$

where $k$ is the wavenumber; $\eta$ is the free space wave impedance; $\beta$ is the phase constant; $\lambda$ is the wavelength in free space; $\lambda_{c n}$ is the cutoff wavelength of the dominant 


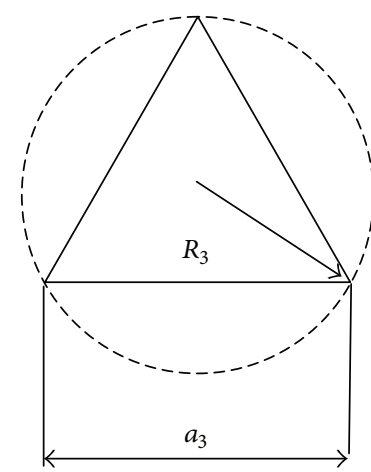

(a)

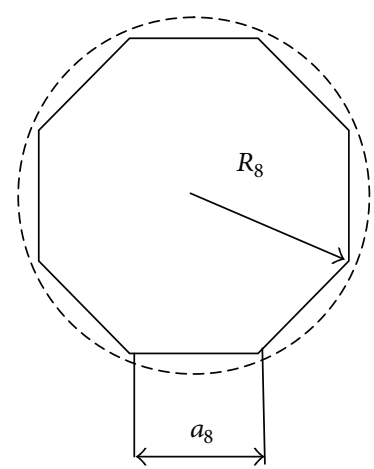

(b)

FIGURE 1: Triangular (a) and octagonal (b) waveguides.

mode. The eigenvalues of RPW can be calculated using the results of numerical modeling obtained with the help of the finite element method (FEM) implemented in PDE Toolbox of MATLAB software [13]. Longitudinal formulation of $2 \mathrm{D}$ Helmholtz equation with Neumann boundary conditions was utilized in finite element modeling of the lowest TE-mode of each RPW. Automated mesh generator and adaptive mesh refiner based on Rosenberg-Stenger scheme are realized in PDE Toolbox for flexible triangulation of arbitrary shaped 2D domains. Electromagnetic field components were approximated by the first-order polynomial functions and generalized eigenvalue problem was solved by Arnoldi algorithm. Number of triangular elements in the mesh for all RPW did not exceed 40,000. Preliminary testing of numerical solution on examples of square $(n=4)$ and cylindrical $(n \geq 32)$ waveguides has shown a good agreement with analytical solutions for these classical transmission lines available from the literature. Additional details of RPW simulations by means of the FEM are described in $[12,13]$.

Curve-fitting procedures of MATLAB and MS Excel have been applied to derive a closed-form expressions using numerically obtained dependencies $\lambda_{c n}(n)$, where $3 \leq n \leq$ 100:

$$
\begin{aligned}
& \lambda_{c n}=(0.5529 n-0.1863) a_{n}, \quad 3 \leq n \leq 8, \\
\lambda_{c n}= & \left(0.0019 n^{3}-0.0555 n^{2}+0.5561 n+1.3777\right) R_{n} \\
= & q R_{n}, \quad 3 \leq n \leq 8, \\
& \lambda_{c n}=(0.0018 n+3.2362) R_{n}, \quad 8 \leq n \leq 100 .
\end{aligned}
$$

Determination coefficient for (3)-(5) was $R^{2} \geq 0.9998$.

\section{Numerical Verification}

Numerical simulation tool-commercial software QuickWave $3 D$ [14] based on the finite difference time domain (FDTD) method-has been utilized in this study for checking (4). Comparative analysis of two numerical techniques, FEM and FDTD, and analytical approach described in [10] are given in Table 1.
TABLE 1: Normalized cutoff wavelengths of the lowest TE-mode of RPW.

\begin{tabular}{cccc}
\hline$n$ & \multicolumn{3}{c}{$\lambda_{c n} / R_{n}$} \\
FDTD & Anal. [10] \\
\hline 3 & FEM & 2.587 & 2.275 \\
4 & 2.599 & 2.83 & 2.828 \\
5 & 2.828 & 3.014 & 3.053 \\
6 & 3.0155 & 3.128 & 3.168 \\
7 & 3.127 & 3.2 & 3.235 \\
8 & 3.2 & 3.251 & 3.273 \\
\hline
\end{tabular}

Junctions of RPW can be successfully employed for design of microwave transitions from widely available coaxial line to RPW. One of such approaches is analyzed in present study.

Let us consider standard cylindrical waveguide (CW) with radius $R=4.181 \mathrm{~cm}$ and the $\mathrm{TE}_{11}$-mode propagating at ISM-frequency $2.45 \mathrm{GHz}$. Matching of this waveguide with RPW is achieved when

$$
S_{11}=\frac{\widetilde{Z}-Z_{n}^{\mathrm{TE}}}{\widetilde{Z}+Z_{n}^{\mathrm{TE}}} \longrightarrow \min
$$

where $S_{11}$ is the reflection coefficient; $\widetilde{Z}$ is the wave impedance of the $\mathrm{TE}_{11}$-mode of $\mathrm{CW}$ at $2.45 \mathrm{GHz}$ and according to (2), $\widetilde{Z}=733.37 \Omega$.

Condition (6) will be satisfied if $Z_{n}^{\mathrm{TE}} \rightarrow \widetilde{Z}$. Then, in order to find sizes of RPW which correspond to (6) we can rewrite (2) as

$$
\lambda_{c n}=\frac{\lambda}{\sqrt{1-(120 \pi / \widetilde{Z})^{2}}} .
$$

And now employing (4) we obtain

$$
R_{n}=\frac{\lambda}{q \sqrt{1-(120 \pi / \widetilde{Z})^{2}}} .
$$




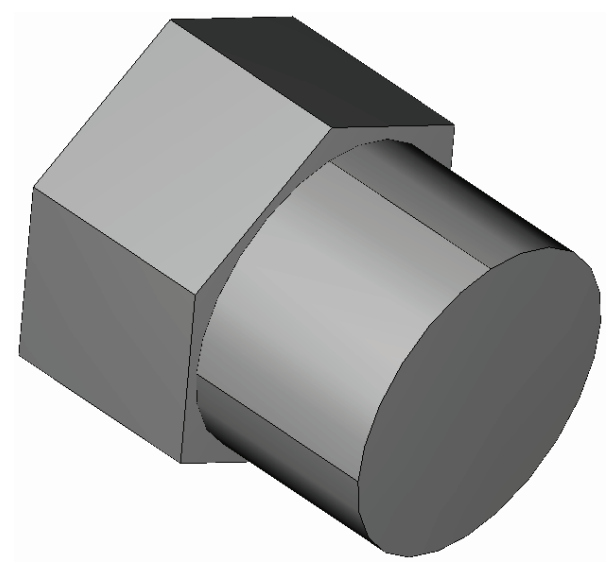

FIGURE 2: Junction of cylindrical and hexagonal waveguides.

TABLE 2: Reflection coefficient of waveguide junctions.

\begin{tabular}{lcccccc}
\hline$n$ & 3 & 4 & 5 & 6 & 7 & 8 \\
\hline$R_{n}, \mathrm{~cm}$ & 5,488 & 5,045 & 4,732 & 4,563 & 4,459 & 4,390 \\
$\left|S_{11}\right|$ & 0.251 & 0.062 & 0.024 & 0.012 & 0.0076 & 0.005 \\
\hline
\end{tabular}

Six 3D FDTD models of CW-RPW junctions for $3 \leq n \leq$ 8 have been built applying QuickWave 3D and (8). One of such models is shown in Figure 2.

Input signal in the form of a pulse of spectrum from $2.3 \mathrm{GHz}$ to $2.6 \mathrm{GHz}$ and $\mathrm{TE}_{11}$-mode was selected for $\mathrm{CW}$. Analogous signal but for arbitrary shaped transmission line was used in the output port. Simulation results: absolute values of reflection coefficient $\left|S_{11}\right|$ at $2.45 \mathrm{GHz}$ are listed in Table 2.

Obtained data demonstrate that proposed approach to the RPW wave impedance definition works well when $n \geq$ 4. For the $n=3,\left|S_{11}\right|$ value is higher than expected. Additional calculations have shown that matching condition (6) is satisfied for $R_{3}=4.9 \mathrm{~cm}$ and $\left|S_{11}\right|=0.115$.

\section{Example of Microwave Transition Design}

Analytical approach described in present study has been used for building the model of coaxial waveguide transition shown in Figure 3. Transition is consisted of two sections: coaxial square waveguide (SQW) adapter and SQW-CW junction. Coaxial line with sizes $D=7 \mathrm{~mm}$ and $d=3.04 \mathrm{~mm}$ excites $\mathrm{TE}_{10}$ mode in SQW $100 \mathrm{~mm}$ length with side wall size $a_{4}=$ $71.347 \mathrm{~mm}$. The dominant mode of SQW is transformed in $\mathrm{TE}_{11}$-mode of CW $15 \mathrm{~mm}$ length and $83.62 \mathrm{~mm}$ diameter in waveguide junction.

Cylindrical metal probe $t=2 \mathrm{~mm}$ connected to the coaxial feeder is placed at the distance $w=30 \mathrm{~mm}$ from short circuited wall of SQW. Optimization problem solution has allowed determining the probe height value $s=25 \mathrm{~mm}$.

Results of numerical modeling: absolute values of reflection coefficient in frequency range $2.2 \leq f, \mathrm{GHz} \leq 2.7$ are represented in Figure 4. FDTD model has been verified by 3D FEM model developed with the help of COMSOL software

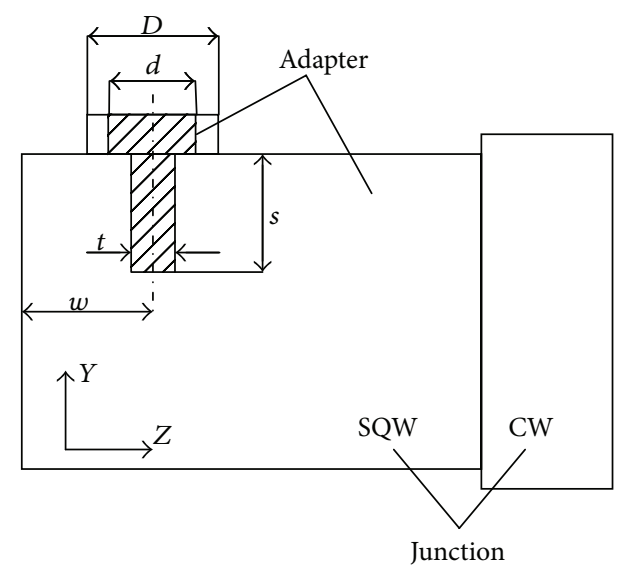

Figure 3: Configuration of microwave transition under study.

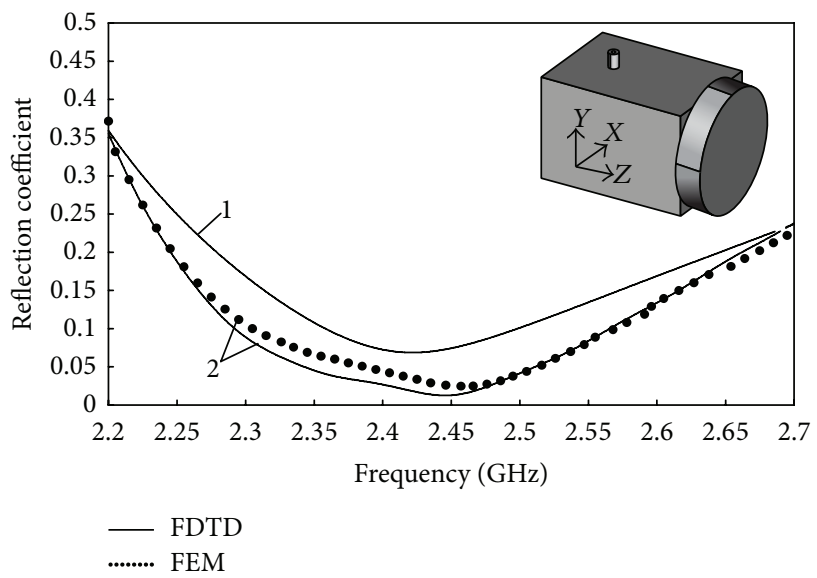

FIGURE 4: Absolute values of reflection coefficient for microwave coaxial SQW adapter (1) and complete transition coaxial SQW-CW (2).

[15]. Both techniques agree well near the ISM-frequency $2.45 \mathrm{GHz}$.

And now taking into account results in Table 2, RPW with any number of side walls can be used instead of CW to design transition from coaxial line to the selected RPW.

\section{Conclusion}

It has been proved that the classical approach to the wave impedance definition previously applied for rectangular and cylindrical waveguides is also applicable for RPW. Exception observed for the triangular waveguide should be a subject of a separate study. Obtained data can be employed in design of various microwave components, for example, transitions, on RPW.

\section{Conflict of Interests}

The author declares that there is no conflict of interests regarding the publication of this paper. 


\section{References}

[1] J. M. Simeoni and M. Jofre, "Equilateral triangular waveguide antenna-a spectral domain analysis," IET Microwaves, Antennas and Propagation, vol. 4, no. 3, Article ID IMAPCH000004000003000296000001, pp. 296-304, 2010.

[2] J.-H. Hwang and Y. Oh, "Compact orthomode transducer using single-ridged triangular waveguides," IEEE Microwave and Wireless Components Letters, vol. 21, no. 8, pp. 412-414, 2011.

[3] Y. Tao, Z. Shen, and G. Liu, "Closed-form expressions for the equivalent circuit model of square-waveguide T-junctions and its application in ortho-mode transducer design," IEEE Transactions on Microwave Theory and Techniques, vol. 58, no. 5, pp. 1167-1174, 2010.

[4] A. Mediavilla, J. L. Cano, and K. Cepero, "On the octave bandwidth properties of octagonal-shaped waveguide mode transformers," IEEE Transactions on Microwave Theory and Techniques, vol. 59, no. 10, pp. 2447-2451, 2011.

[5] V. N. Tran, "An applicator design for processing large quantities of dielectric materials," in Proceedings of the Symposium on Microwave: Theory and Applications in Material Processing, pp. 683-690, Cincinnati, Ohio, USA, 1991.

[6] V. S. Il'in, S. N. Il'in, and D. A. Usanov, "Properties of an orthogonal-turnstile junction of three square waveguides and a hexagonal waveguide," Journal of Communications Technology and Electronics, vol. 42, no. 2, pp. 121-126, 1997.

[7] C. Y. Wang, "Exact solution of equilateral triangular waveguide," Electronics Letters, vol. 46, no. 13, pp. 925-927, 2010.

[8] C. Y. Wang, "Cutoff frequencies of perturbed circular waveguides and polygonal waveguides," Journal of Electromagnetic Waves and Applications, vol. 16, no. 2, pp. 151-158, 2002.

[9] M. Lucido, G. Panariello, and F. Schettino, "Full wave analysis of arbitrary polygonal section waveguides," in Proceedings of the IEEE MTT-S International Microwave Symposium (IMS '07), pp. 1675-1678, Honolulu, Hawaii, USA, June 2007.

[10] B. Ravelo and A. K. Jastrzebski, "EM parameters of regular polygonal waveguide," in Proceedings of the 14th European Microwave Conference (EuMC '11), pp. 786-789, Manchester, UK, October 2011.

[11] Q. Zheng, 1. Ma, and J. Li, "A simple estimate for cutoff number of lowest order TM mode of hollow metallic waveguide of arbitrary cross section," International Journal of RF and Microwave Computer-Aided Engineering, vol. 10, no. 3, pp. 159$163,2000$.

[12] V. V. Komarov, "Eigenmodes of regular polygonal waveguides," Journal of Infrared, Millimeter, and Terahertz Waves, vol. 32, no. 1, pp. 40-46, 2011.

[13] MathWorks, PDE Tootbox, MATLAB, Natick, Mass, USA, 2013, http://www.mathworks.com/.

[14] QWED, QuickWave-3D, QWED, Warsaw, Poland, 2013, http:// www.qwed.com.pl/.

[15] COMSOL Multiphysics V.4.3, 2013, http://www.comsol.com/. 

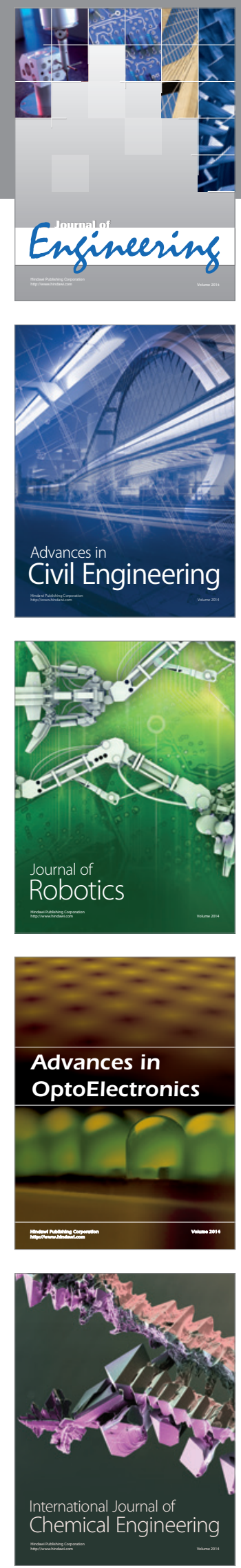

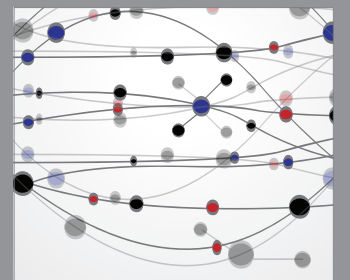

The Scientific World Journal
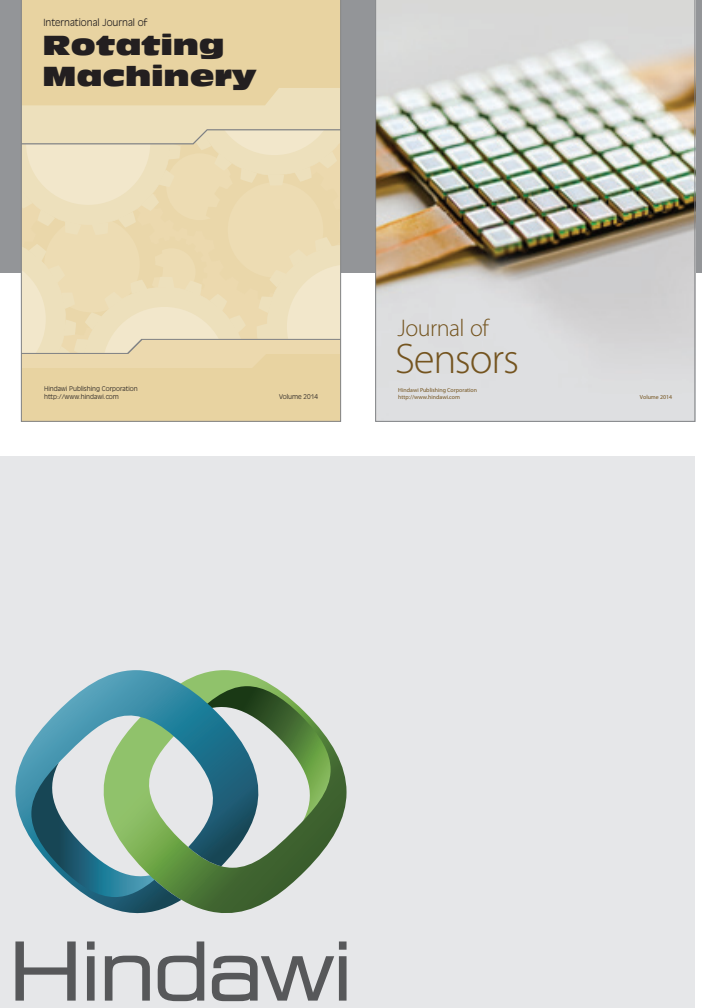

Submit your manuscripts at http://www.hindawi.com
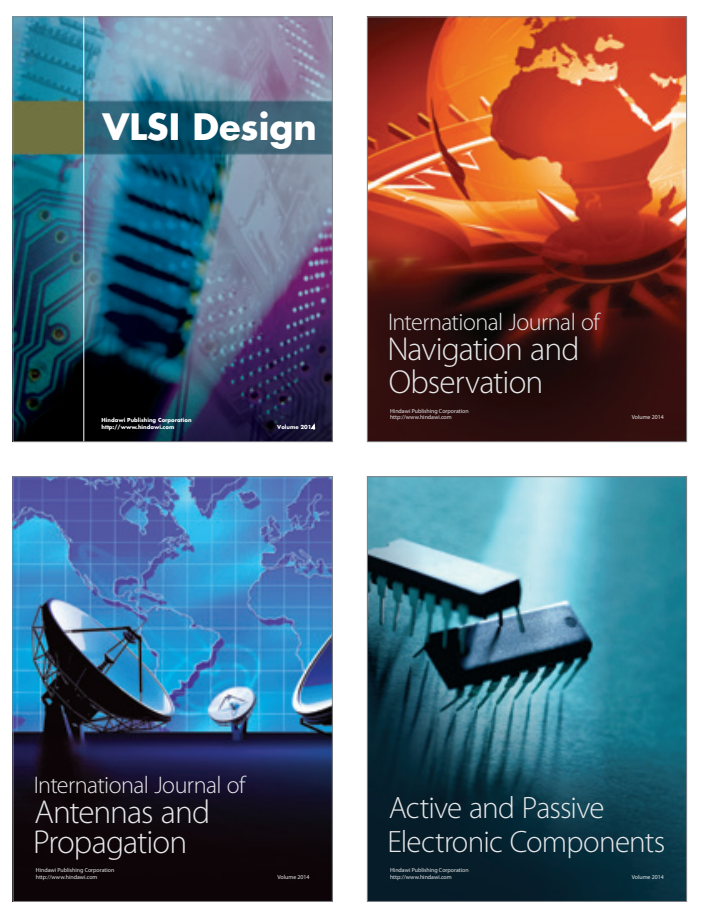
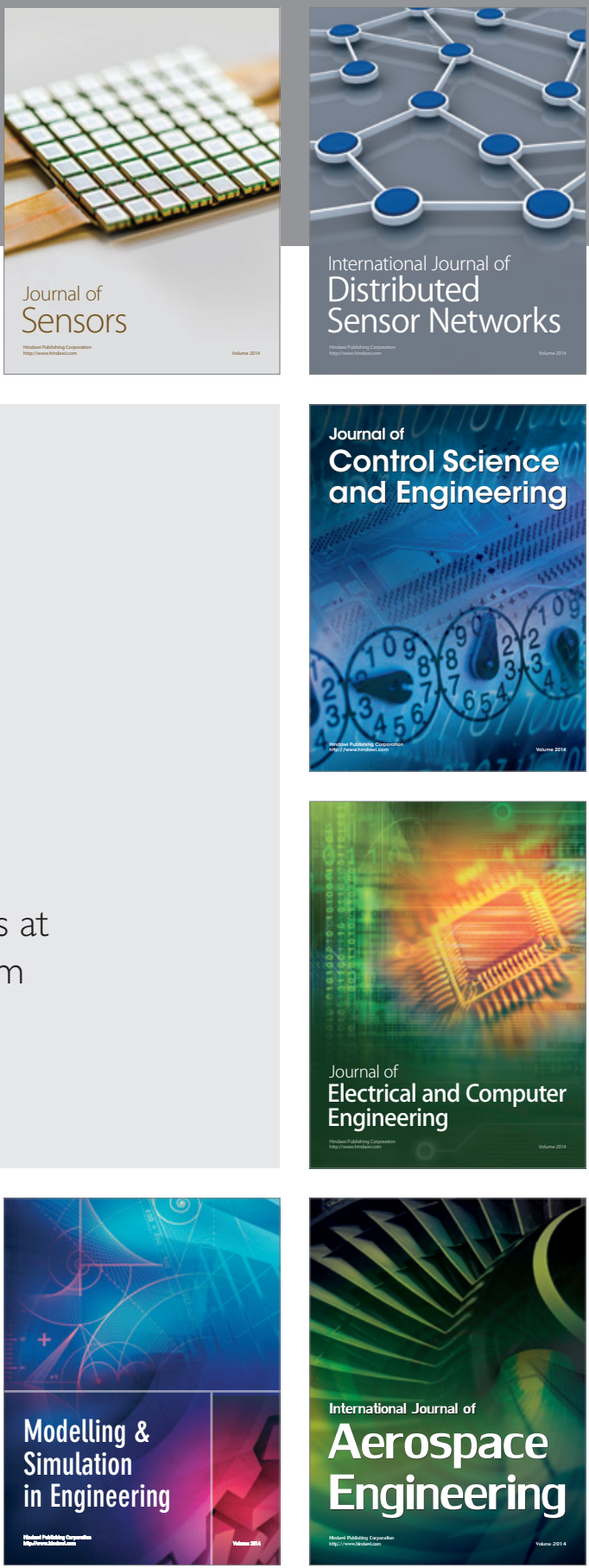

Journal of

Control Science

and Engineering
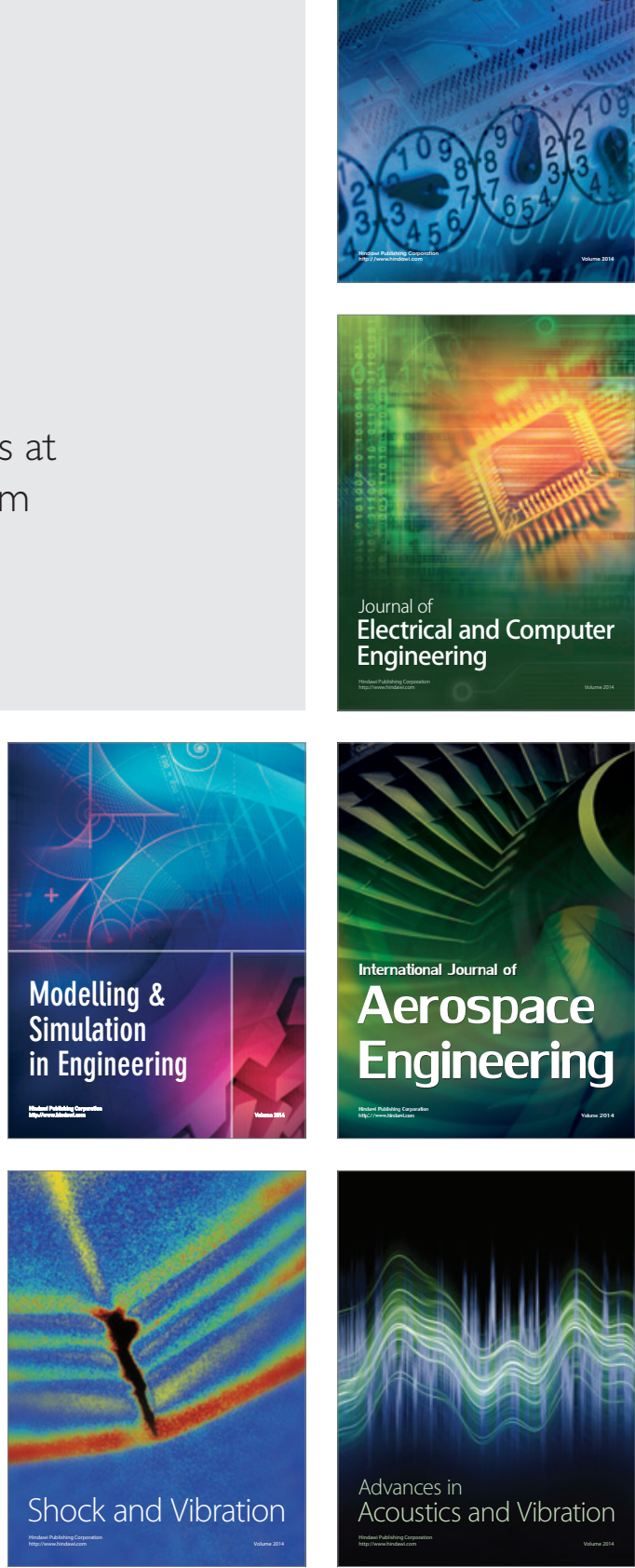